\title{
Behavioral and neural toxicity of the artemisinin antimalarial, arteether, but not artesunate and artelinate, in rats
}

\author{
Raymond F. Genovese ${ }^{\mathrm{a}, *}$, Donald B. Newman ${ }^{\mathrm{b}}$, Thomas G. Brewer ${ }^{\mathrm{c}}$ \\ ${ }^{a}$ Division of Neurosciences, Walter Reed Army Institute of Research, 503 Robert Grant Avenue, Silver Spring, MD 20910-7500, USA \\ ${ }^{\mathrm{b}}$ Department of Anatomy and Cell Biology, Uniformed Services University of the Health Sciences, Bethesda, MD 20814-4799, USA \\ ${ }^{\mathrm{c}}$ Armed Forces Institute of Medical Sciences, Bangkok 10400, Thailand
}

Received 23 August 1999; received in revised form 8 March 2000; accepted 23 March 2000

\begin{abstract}
Three artemisinin antimalarials, arteether (AE), artesunate (AS), and artelinate (AL) were evaluated in rats using an auditory discrimination task (ADT) and neurohistology. After rats were trained on the ADT, equimolar doses of $\mathrm{AE}(25 \mathrm{mg} / \mathrm{kg}$, in sesame oil, $n=6)$, AS $(31 \mathrm{mg} / \mathrm{kg}$, in sodium carbonate, $n=6)$, and $\mathrm{AL}(36 \mathrm{mg} / \mathrm{kg}$, in saline, $n=6)$, or vehicle (sodium carbonate, $n=6)$ were administered (IM) for 7 consecutive days. Behavioral performance was evaluated, during daily sessions, before, during, and after administration. Histological evaluation of the brains was performed using thionine staining, and damaged cells were counted in specific brainstem nuclei of all rats. Behavioral performance was not significantly affected in any rats treated with AS, AL, or vehicle. Furthermore, histological examination of the brains of rats treated with AS, AL, and vehicle did not show damage. In stark contrast, all rats treated with AE showed a progressive and severe decline in performance on the ADT. The deficit was characterized by decreases in accuracy, increases in response time and, eventually, response suppression. When performance on the ADT was suppressed, rats also showed gross behavioral signs of toxicity that included tremor, gait disturbances, and lethargy. Subsequent histological assessment of AE-treated rats revealed marked damage in the brainstem nuclei, ruber, superior olive, trapezoideus, and inferior vestibular. The damage included chromatolysis, necrosis, and gliosis. These results demonstrate distinct differences in the ability of artemisinins to produce neurotoxicity. Further research is needed to uncover pharmacokinetic and metabolic differences in artemisinins that may predict neurotoxic potential. (C) 2000 Elsevier Science Inc. All rights reserved.
\end{abstract}

Keywords: Antimalarial; Artemisinin; Neurotoxicity; Behavior; Safety assessment; Rats

\section{Introduction}

Derivatives of artemisinin (qinghaosu) [12] the active principal of the Chinese herb Artemisia annua L., are currently in use, and under development, as potent antimalarials [6]. The therapeutic value of artemisinins is particularly attractive as the occurrence of multidrug resistant strains of Plasmodium falciparum increases. That is, artemisinins have been successfully introduced into treatment regimens in areas where the effectiveness of existing chemotherapy (e.g., chloroquine, sulfadoxine-pyrimethamine, mefloquine) has decreased [30].

* Corresponding author. Tel.: +1-301-319-9721; fax: +1-301-3199905.

E-mail address: raymond.genovese@na.amedd.army.mil (R.F. Genovese).
There is a paucity of clinical studies that have specifically investigated the neurotoxic potential of the several varieties of artemisinins available. It is, however, generally believed that the class of compounds is well tolerated, and has a wide safety margin (e.g., Ref. [25]). On the other hand, the artemisinins, arteether (AE), and artemether have been shown to produce a somewhat selective pattern of brainstem neurotoxicity in rats, dogs, and Rhesus monkeys $[3,4,8-11,23]$. For example, we have found that repeated [9-11] or single [8] administration of $\beta-\mathrm{AE}$, in rats, produces significant and progressive neuropathology, including chromatolysis and gliosis, in brainstem nuclei associated with auditory, vestibular, and muscular control functions. Furthermore, AE-induced neurotoxicity is difficult to detect behaviorally, particularly when mild. We have, however, previously reported that performance on an auditory discrimination task 
(ADT) is degraded as a reflection of $\mathrm{AE}$ administration [10], and thus, can serve as a behavioral index of toxicity. Performance on the ADT may be degraded as a reflection of AE-induced neurotoxicity, in part, because of the vulnerability of two brainstem auditory nuclei, the superior olive (consisting of medial and lateral subdivisions), and the nucleus of the trapezoid body $[8,10]$. The mechanism of action of artemisinin-induced neurotoxicity is unknown although a prominent role has been speculated for the formation of free radicals from the endoperoxide structure and the function of specific protein alkylation (see Refs.[20,21]).

In the present study, we further investigated artemisinin-induced neurotoxicity in rats. Specifically, we were interested in comparing the potential neurotoxic effects of several artemisinins. Thus, we evaluated the effects of repeated administration of three artemisinins, AE, artesunate ( $\mathrm{AE})$, and artelinate ( $\mathrm{AL}$ ), and vehicle, using the $\mathrm{ADT}$ and subsequent histopathology. $\beta-\mathrm{AE}$, is the ethyl ether analogue of dihydroartemisinin (DHA), has substantial antimalarial activity [26], and is currently undergoing advanced clinical development [5,13,22]. $\alpha$-Artesunic acid (AS) is, perhaps, the artemisinin most widely employed clinically and is a very effective antimalarial (e.g., Refs. $[19,24])$. AS is frequently administered in combination with other therapeutic agents [7,29]. Sodium AL [18] is an artemisinin currently under development that, while not yet used in a clinical setting, has been shown to be an effective antimalarial agent in mice [27] and Rhesus monkeys [28].

The three artemisinins used in the present study could be distinguished by several characteristics. AS and AE are comparable in that both compounds are predominantly metabolized to DHA, which has potent antimalarial qualities (cf. Ref. [6]). In this respect, a somewhat larger percentage of AS, compared to $\mathrm{AE}$, is metabolized to DHA (e.g., Ref. [17]). They differ in that AE is lipidsoluble, whereas AS is water-soluble. Furthermore, AE has a relatively long elimination half-life compared to other artemisinins, especially AS (e.g., Refs. $[1,6,13])$. AL is similar to AS in that it is also water-soluble. $\mathrm{AL}$, however, differs from both $\mathrm{AS}$ and $\mathrm{AE}$ in that only a very small percentage is metabolized to DHA $[16,17]$.

We chose an administration regimen for AE $(25 \mathrm{mg} /$ $\mathrm{kg} /$ day $\times 7$ ) and route of administration (IM) that has previously been shown to produce profound behavioral and neural toxicity $[8,10]$. Equimolar doses of AS and AL were administered using the same regimen. For behavioral assessment, rats were trained on the ADT, and performance was evaluated before, during, and after drug administration. Subsequently, histological assessment focused on select brainstem nuclei (trapezoideus, superior olive, ruber, inferior vestibular). These nuclei were chosen for examination because they have previously been shown to be particularly vulnerable to artemisinin neurotoxicity $[3,8-10,12]$.

\section{Materials and methods}

\subsection{Subjects}

Adult male Sprague-Dawley rats (Charles River, Wilmington, MA) were used. Rats were individually housed under a 12-L:12-D cycle (lights on at $0600 \mathrm{~h}$ ), and water was always available in the home cages. Food was restricted to that earned during behavioral sessions and supplemental feedings (Agway Pro Lab Rodent Chow) to maintain body weights at approximately $320 \mathrm{~g}$.

\subsection{Apparatuses}

Behavioral testing was conducted using standard rodent operant conditioning chambers (Coulbourn Instruments, Allentown, PA, Model E-10-10 or equivalent). Each chamber contained two fixed response levers, a speaker module, stimulus lights mounted above each response lever, and a food dispenser to deliver $45 \mathrm{mg}$ of food pellets (BioServe, Frenchtown, NJ). Chambers were housed in ventilated, sound - and light-attenuating cubicles. The speaker in each chamber was attached to a mixer/amplifier (Coulbourn Instruments, Model S82 -24) with inputs from sound generators that produced white noise $(1 \mathrm{~Hz}-10 \mathrm{kHz})$ (Coulbourn Instruments, Model S81-02) or precision sine waves (Coulbourn Instruments, Model S81 -06). Sound pressure level (SPL re 20 $\mu \mathrm{Pa}$ ) was determined with a sound level meter [(A) scaling] (Edmund Scientific, Model N38732) and was measured inside the operant chamber at a fixed point approximately $2 \mathrm{in}$. from the front of the speaker panel. Experimental events were controlled and monitored using a PDP - 11/73 microcomputer (Digital Equipment, Maynard, MA), using the SKED (State Systems, Kalamazoo, MI) control language.

\subsection{Behavioral procedure}

Details of the ADT appear elsewhere [10]. Briefly, rats were trained on a two-choice, discrete-trial ADT where the discriminative stimuli were white noise $(70 \mathrm{~dB})$ and a sine wave tone $(750 \mathrm{~Hz}$ at $75 \mathrm{~dB})+$ white noise $(70 \mathrm{~dB})$. Each session lasted approximately $60 \mathrm{~min}$, and consisted of a series of discrete trials, during which a discriminative stimulus (either white noise or tone + white noise) was presented. Correct choices produced food reinforcement and incorrect choices delayed the next choice opportunity (i.e., the presentation of another discriminative stimulus). Both stimulus lights (located above the response levers) were illuminated upon presentation of the auditory stimulus, and both remained on for $10 \mathrm{~s}$ or until a choice was made. Trial types (i.e., noise or tone + noise) were randomly determined with an equal probability of presentation of each discriminative stimulus. If no choice was made within the presentation period, the trial was terminated and scored as a "no decision." The houselight and stimulus lights were extinguished upon termination of the stimulus presentation period. If the choice response was 
correct (i.e., a left lever press during presentation of the noise alone or a right lever press during presentation of the tone + noise) then food (a single $45 \mathrm{mg}$ pellet) was delivered. Stimulus presentation periods were separated by an intertrial interval (ITI), which was $20 \mathrm{~s}$ unless preceded by an incorrect choice, in which case it was $60 \mathrm{~s}$. Lever presses during the ITI were recorded but had no programmed consequences except that lever presses during the last $3 \mathrm{~s}$ of an ITI extended the duration of the ITI by $3 \mathrm{~s}$. Behavioral sessions were normally conducted Monday-Friday, or daily during drug administration phases.

Stable performance was judged to occur if overall accuracy was approximately $80 \%$ or better, and less than approximately $15 \%$ deviation in overall correct responding was observed for at least seven consecutive sessions.

\subsection{Pharmacological procedure}

$\beta-\mathrm{AE}(\mathrm{MW}=312), \alpha-\mathrm{AS}(\mathrm{MW}=384)$, and sodium AL $(\mathrm{MW}=441)$ were obtained under contract to the US Army (Starks Associates, NY). AE was dissolved in sterile sesame oil. AL was dissolved in $0.9 \%$ sterile saline. AS was dissolved in $2.5 \%$ sodium carbonate solution (i.e., AS), and a $2.5 \%$ sodium carbonate solution was used for all vehicle injections. We chose to inject the sodium carbonate solution as a vehicle because sesame oil vehicle injections were shown to have no effects previously (e.g., Ref. [10]). When responding on the ADT was stable, rats were assigned to one of four groups ( $n=6$ for each group) (vehicle, AE - $25 \mathrm{mg} / \mathrm{kg} /$ day, AS - $31 \mathrm{mg} / \mathrm{kg} /$ day, and $\mathrm{AL}-36 \mathrm{mg} / \mathrm{kg} /$ day), matched on the basis of overall choice accuracy. Injections were administered (approximately $75 \mathrm{~min}$ before behavioral sessions) daily for 7 consecutive days. Drug and vehicle injections were given IM into the thigh muscle in a volume of $0.5 \mathrm{ml} / \mathrm{kg}$ body weight, using a 28 -gauge needle. Daily behavioral sessions were continued for 7 additional days (i.e., postdrug days) until rats were euthanized for histopathological analysis on the 14th day following the first injection. We employed a postdrug interval to maximize the detection of neural and behavioral changes that might occur after drug administration had been discontinued.
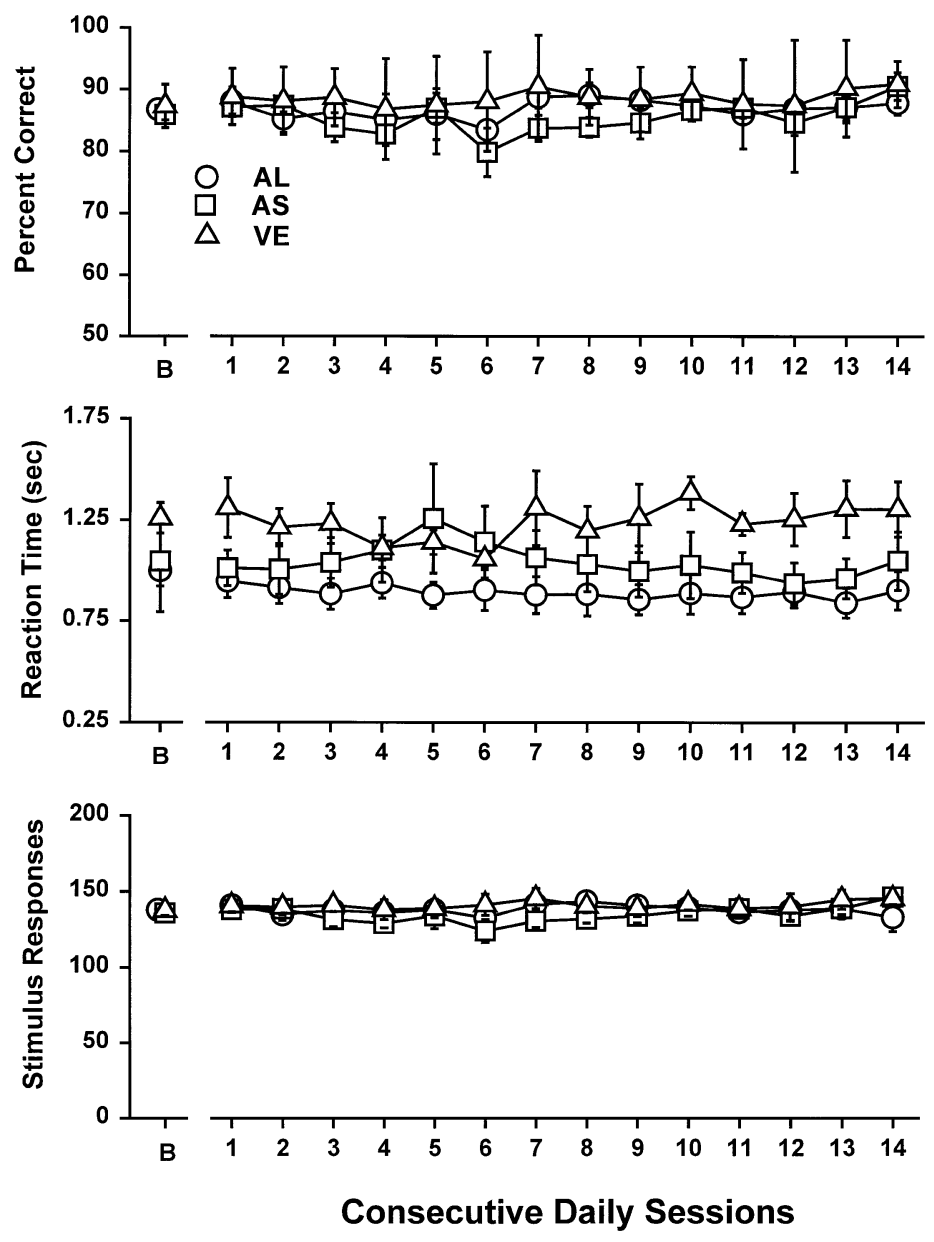

Fig. 1. Performance of rats on the ADT receiving injections of AS ( $31 \mathrm{mg} / \mathrm{kg} /$ day $\times 7)$, AL ( $36 \mathrm{mg} / \mathrm{kg} / \mathrm{day} \times 7)$, or vehicle, for percent correct (top), reaction time (middle), and the number of stimulus responses (bottom). Injections were given before sessions $1-7$. Each point represents the mean of six rats. Vertical lines about each bar represent the SEM. Points above B represent average performance measures for the seven sessions preceding injections. 


\subsection{Histological procedure}

Rats were deeply anesthetized with sodium pentobarbital (50 mg, IP) and perfused intracardially with a heparinized buffered saline rinse followed by at least $100 \mathrm{ml}$ of Bouin's fixative for $24 \mathrm{~h}$. Brains were then removed from the animals' skulls, and stored for 1 week in $70 \% \mathrm{EtOH}$ under refrigeration. Selected brain sections were cut based upon earlier studies $[8-10]$ that determined the areas most vulnerable to artemisinin damage. Specifically, two blocks were taken from each brain; one encompassing the midbrain, the other encompassing the caudal pons and rostral medulla (as well as the cerebellum). The blocks were embedded in paraffin and sectioned transversely at a thickness of $20 \mu \mathrm{m}$. Twelve serial sections were taken from the midbrain block. Twelve serial sections were taken from the rostral end of the pons-medulla block, which was then inverted, and 12 serial sections cut from its caudal end. Thus, a total of 36 sections were taken from each rat's brainstem. The sections were mounted on glass slides, three per slide, stained with Cresyl violet, and coverslipped with Permount.

For each brain, cell counts were made on five select brainstem nuclei: the nucleus of the trapezoid body (n. trap.), the red nucleus (n. ruber), the superior olivary nucleus (n. sup. olive), the inferior vestibular nucleus (n. inf. vest.), and the facial motor nucleus (n. facialis). The first four of these nuclei have been shown to be especially vulnerable to the effects of artemisinin-derived compounds. The facial motor nucleus, on the other hand, has been shown to be particularly resistant to the neurotoxic effects of artemisinins, and, therefore, served as an internal control. For each nucleus analyzed, cell counts were done on six serial sections.

To avoid counting split neurons, a given nucleus was counted on alternate sides from one section to the next. In each section, four adjacent fields of neurons in a given nucleus were counted at an eyepiece magnification of $640 \times$ using a Zeiss microscope equipped with a net reticle. Using a hand counter, the cells were scored as being either
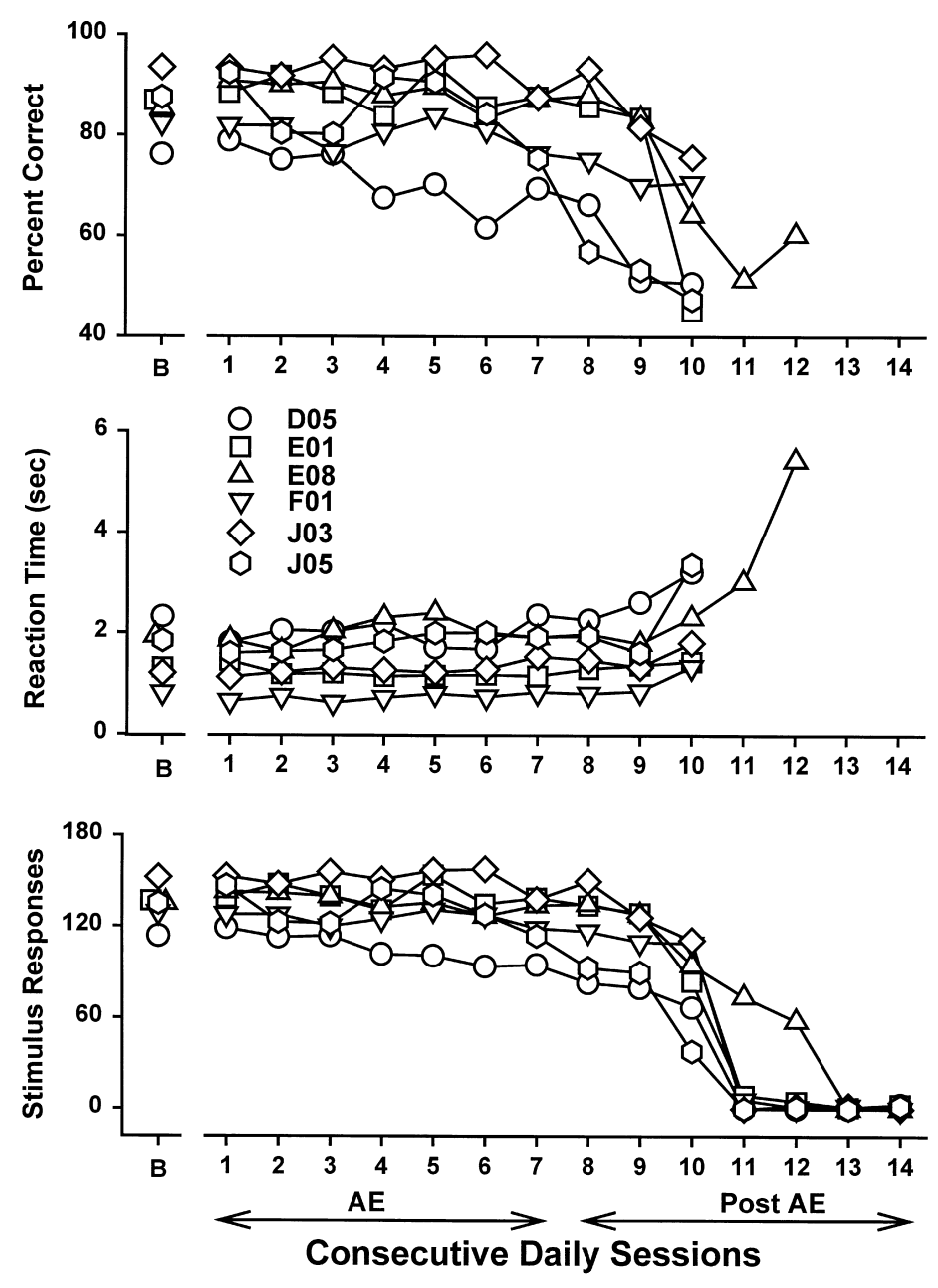

Fig. 2. Performance of rats on the ADT receiving injections of $\mathrm{AE}(25 \mathrm{mg} / \mathrm{kg} /$ day $\times 7)$ for percent correct (top), reaction time (middle), and the number of stimulus responses (bottom). Injections were given before sessions 1-7. Each point represents performance of a single rat. Points above B represent average performance measures for the seven sessions preceding injections. 
damaged or undamaged. Neurons exhibiting any of the following distinguishing characteristics were scored as damaged: (1) swollen cell body (perikaryon); (2) dissolution of Nissl substance (chromatolysis); (3) condensation of the Nissl substance against the nuclear membrane; (4) nuclear eccentricity; (5) crenelated nuclear membrane; (6) swollen nucleolus; (7) nuclear shrinkage. To further avoid the problem of split neurons, only neurons exhibiting a distinct nucleolus were counted. A minimum of 100 neurons were scored for each nucleus analyzed (cell counts usually totaled between 200 and 300 cells/nucleus). Cell count data were then expressed as the percentage of the total nuclear sample analyzed exhibiting damage.

\subsection{Data analysis}

When a response or an experimental event occurred under the ADT, the elapsed time within the session was recorded. From these data, measures of overall percent correct (i.e., correct choices/incorrect choices + correct choices), average response time (i.e., the time from the onset of the auditory stimulus to a choice response), and the total number of responses to the stimuli, for each session, were calculated. When fewer than 10 stimulus responses occurred during any session, however, the measures of percent correct and reaction time were not used. The latter outcome was only observed in $\mathrm{AE}$-treated rats and only during the last four sessions. To statistically evaluate behavioral performance under the ADT, repeated-measures ANOVA (SAS statistical software, Cary, NC) were used to assess the differences in percent correct, reaction time, and number of stimulus responses, during the drug and postdrug sessions for all the four treatment groups. Statistical significance was designated if $p<0.05$. In the case of AE-treated rats, however, responding was suppressed during some sessions such that reaction time and percent correct could not be calculated. In the latter instances, ANOVA was not calculated.

To statistically evaluate the difference between percent damaged neurons for the quantitative analysis of selected nuclei, ANOVA were calculated using the General Linear Models procedure of the SAS statistical software package (SAS statistical software). When a significant $F$-value was obtained for a main effect in any of the nuclei, multiple comparison tests were conducted using Dunnett's $t$, which compared the percentages of affected cells in each dose condition to the vehicle condition. Statistical significance was designated if $p<0.05$.

\section{Results}

All rats appeared normal and healthy throughout the duration of the experiment with the exception of rats treated with AE. During the last several days of the study the latter rats exhibited motor deficits, gait disturbances, tremors, and weight loss.

\subsection{Behavioral performance}

During baseline sessions, performance under the ADT was, in general, stable in all rats, and typically overall percent correct was above $80 \%$. Response time was relatively fast (typically $0.75-1.25 \mathrm{~s}$ ) and stable. The number of responses to stimuli was also relatively stable (typically $100-150$ responses per session) and typically $95-125$ food pellets were presented during a session (see Figs. 1 and 2, points above B). Typically, 100-200 training sessions were required to establish baseline performance.

Vehicle, AS, and AL treatments did not have observable effects on any of the performance measures of the ADT (see Fig. 1). Performance remained stable during and after daily administration of vehicle and the artemisinins, AS, and AL. ANOVA revealed no statistically significant effects for measures of percent correct, reaction time, and total number of responses $(p>0.05)$. In contrast with $\mathrm{AS}$ and $\mathrm{AL}, \mathrm{AE}$

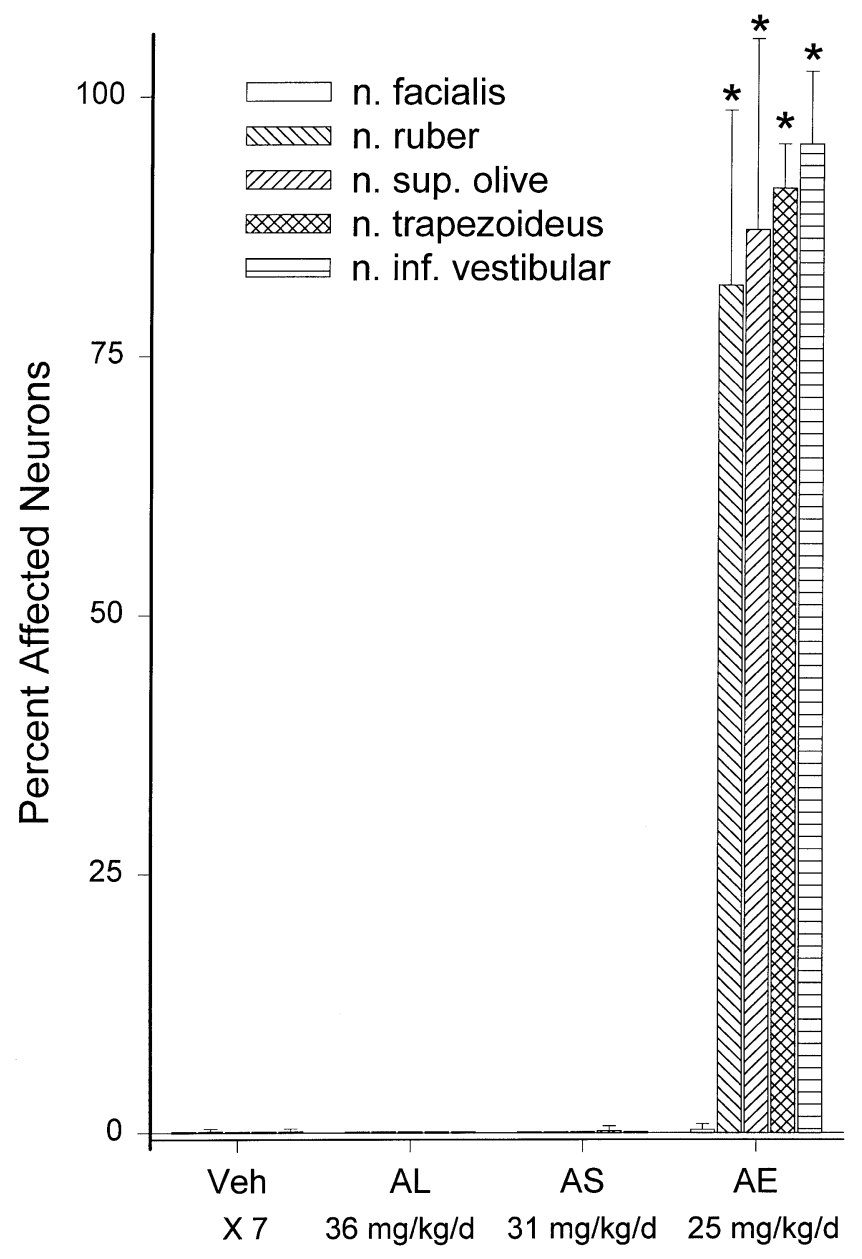

Fig. 3. Average percentage of examined neurons in selected brainstem nuclei judged to be damaged following daily administration of vehicle, AL (36 mg/kg), AS (31 mg/kg), and AE (25 mg/ kg), for 7 days. Rats were sacrificed 7 days following the last administration. Each bar represents mean cell counts from six rats. Vertical lines about each bar represent the SEM. * Indicates a statistically significant difference from the vehicle control $(p<0.05)$. 
administration produced profound performance deficits in all rats. Fig. 2 shows the performance of individual rats, treated with $\mathrm{AE}$, on the ADT. Whereas, performance on the initial days of AE administration was similar to the baseline, performance was progressively disrupted during subsequent sessions. Percent correct (Fig. 2, top) declined in all rats, and the decrease was profound during sessions 9-11. Reaction time (Fig. 2, middle) tended to increase. The number of responses to stimuli (Fig. 2, bottom) decreased systematically during and after AE administration. For all rats, responding was completely, or nearly completely, suppressed during sessions 11-14. The differences in stimulus responses during the testing period was significant, $F(13,65)=88.6, p<0.01$. Multiple contrasts comparing sessions $2-14$ with session 1 indicated a statistically significant difference for sessions $9-14, F(1,5) \geq 14.76$, $p<0.05$. Because responding decreased dramatically during the last six sessions, ANOVA was not calculated for the measures of percent correct and reaction time.

\subsection{Histological analysis}

Fig. 3 shows the results of histopathological examination of the five brainstem nuclei in all rats (due to a technical problem, a damaged cell count for the n. ruber in a single rat, treated with AS, could not be calculated). Neuropathology was not observed in any of the brainstem nuclei examined in vehicle-treated rats. Similarly, neuropathology was not observed in the brainstem nuclei of rats treated with $\mathrm{AS}$ or $\mathrm{AL}$. In contrast, rats treated with $\mathrm{AE}$ showed substantial neuropathology in all of the brainstem nuclei examined except for the $\mathrm{n}$. facialis. Neuropathology in these nuclei included extensive chromatolysis, pyknosis, and gliosis (see Fig. 4). Statistically significant neuropathology was found for the n. trap., $F(3,20)=2706.67$, $p<0.001$, n. sup. olive, $F(3,20)=134.81, p<0.001$, n. ruber, $F(3,19)=131.31, p<0.001$, and n. inf. vest., $F(3,20)=1116.67, p<0.001$. Neuropathology was not found in the $\mathrm{n}$. facialis, $F(3,20)=2.20, p>0.1$. Multiple

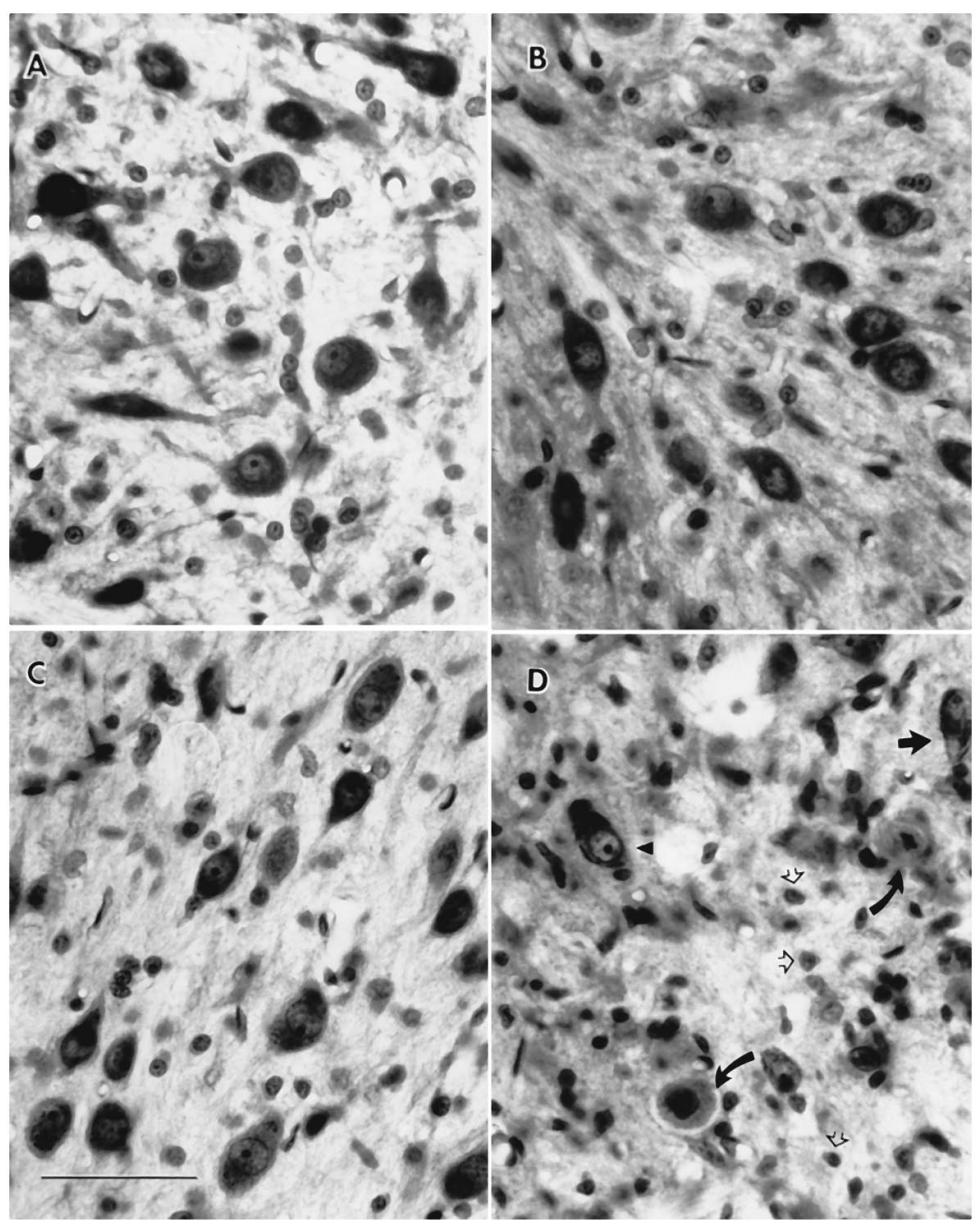

Fig. 4. Photomicrographs illustrating Cresyl violet stained neurons in the superior olive from rats given vehicle $(\mathrm{A}), \mathrm{AS}(31 \mathrm{mg} / \mathrm{kg} / \mathrm{day} \times 7),(\mathrm{B}), \mathrm{AL},(36 \mathrm{mg} /$ $\mathrm{kg} /$ day $\times 7)(\mathrm{C})$, or $\mathrm{AE}(25 \mathrm{mg} / \mathrm{kg} /$ day $\times>7)(\mathrm{D})$. Neurons in rats treated with vehicle, AS, and AL appear unaffected, while neurons from rats receiving AE exhibit substantial damage. While the cell in the upper left appears normal (black arrowhead), other neurons exhibit nuclear eccentricity and chromatolysis (short black arrow), or pyknosis (curved black arrows). Gliosis (open arrows) is also apparent. All four photos are at the same magnification. Scale bar (C) represents $50 \mu \mathrm{m}$. 
contrasts (Dunnett's $t, p<0.05$ ) revealed that all affected nuclei (i.e., n. trap., n. sup. olive, n. inf. vest., and n. ruber) in AE-treated rats showed significant neuropathology compared to damaged cell counts in corresponding nuclei in vehicle control rats. None of the other paired comparisons were statistically significant.

\section{Discussion}

We examined the behavioral and neural toxicity of equimolar dosing regimens of three characteristically different artemisinins and vehicle. Two of the artemisinins, AL and AS, as well as vehicle, had no effects on a behavioral test. Additionally, AL and AS, as well as vehicle, had no neurotoxic effects on examined brainstem nuclei. In stark contrast, AE produced profound behavioral and neural toxicity. It is notable, however, that greater doses, or longer regimens, of AS and AL could have also produced similar toxic effects as we observed with AE. Nonetheless, these results suggest that $\mathrm{AE}$ is a more toxic artemisinin than AS and AL.

AE produced significant performance deficits on the ADT. Initial effects included a decrease in accuracy and an increase in reaction time. A progressive reduction in responding was also observed and eventually, all, or nearly all responding on the ADT was suppressed. It is notable that the behavioral effects of $\mathrm{AE}$ were greater during the latter portion of the administration regimen and during the postadministration testing sessions. Moreover, during the final few sessions of testing, AE-treated rats exhibited gross behavioral signs of toxicity that included lethargy, tremor, and gross gait disturbances. Thus, the onset of behavioral effects was delayed and the deficit was progressive. These results are consistent with the effects of AE obtained previously using the ADT $[8,10]$. AE also produced significant neuropathology in select brainstem nuclei. That is, a large proportion of neurons examined in the n. trap., n. sup. olive, n. ruber, and n. inf. vest. were damaged. Neuropathology was extant and with widespread chromatolysis and necrosis. The profile of brainstem neurotoxicity observed in the present study is very similar to that obtained previously with similar regimens of $\mathrm{AE}$ in rats $[8,10]$, and is consistent with results from studies with dogs and Rhesus monkeys [3,4,23].

Although we did not attempt to measure any pharmacokinetic parameters, it is of interest to consider the role of DHA in the present experiment. DHA is a major metabolite of $\mathrm{AE}$, and produces a similar profile of brainstem neurotoxicity, but at lower doses [2]. Only a very small percentage of AL, which had no neurotoxic effects in the present study, is converted to DHA [16,17]. Thus, DHA may reasonably be implicated as causing the neurotoxicity in the present study. On the other hand, DHA is also the major metabolite of AS, which had no neurotoxic effects in the present study. In fact, $\mathrm{Li}$ et al. [17] reports that an even greater percentage of AS is metabolized to DHA compared with AE. AS, however, is rapidly absorbed, and has a much shorter elimination half-life than $\mathrm{AE}[14,15,17]$. Under comparable administration regimens as in the present study, $\mathrm{AE}$ would be expected to produce a much longer period of DHA activity than AS. Therefore, it is plausible that a sustained level, rather than a peak level, of DHA is the crucial factor leading to neurotoxicity.

The present study demonstrates that equivalent dosing regimens, with respect to molar concentration and route of administration, of different artemisinins, produce dramatically different neurotoxic effects. Thus, the endoperoxide structure, which is the defining characteristic of the artemisinin antimalarial class, and is shared by all three artemisinins used in the present study, is not the sole determinant of neurotoxicity. Further research is, therefore, needed to determine the specific pharmacokinetic and metabolic factors that underlie the mechanism of artemisinin neurotoxicity.

\section{Acknowledgments}

In conducting the research described in this report, the investigators adhere to the "Guide for the Care and Use of Laboratory Animals," as promulgated by the Committee on Care and Use of Laboratory Animals of the Institute of Laboratory Animal Resources, National Research Council. The views of the authors do not purport to reflect the position of the Department of the Army or the Department of Defense (para 4-3, AR 360-5). The authors thank Kimberly A. Gordon for technical help with the conduct of the experiments, and Hong-Anh Nguyen for help with the preparation of the manuscript. This research was supported, in part, by World Health Organization Tropical Disease Research Grants 950304 and 970077.

\section{References}

[1] Benakis A, Schopfer C, Paris M, Plessas ChT, Karayannakos PE, Dondas I, Kotsarelis D, Plessas ST, Skalkeas G. Pharmacokinetics of arteether in dog. Eur J Drug Metab Pharmacokinet 1991;16:325-8.

[2] Brewer TG. Studies to define the sites and mechanisms of arteether/ artemisinin neurotoxicity. Final project report: unpublished (WHO ID\#920560), 1997.

[3] Brewer TG, Grate SJ, Peggins JO, Weina PJ, Petras JM, Levine BS, Heiffer MH, Schuster BG. Fatal neurotoxicity of arteether and artemether. Am J Trop Med Hyg 1994;51:251-9.

[4] Brewer TG, Peggins JO, Grate SJ, Petras JM, Levine BS, Weina PJ, Swearengen J, Heiffer MH, Schuster BG. Neurotoxicity in animals due to arteether and artemether. Trans R Soc Trop Med Hyg 1994;88(1):33-6.

[5] Davidson DE. Role of arteether in the treatment of malaria and plans for further development. Trans R Soc Trop Med Hyg 1994;88(1):51-2.

[6] de Vries PJ, Dien TK. Clinical pharmacology and therapeutic potential of artemisinin and its derivatives in the treatment of malaria. Drugs 1996;52(6):818-36.

[7] Duarte EC, Fontes CJ, Gyorkos TW, Abrahamowicz M. Randomized controlled trial of artesunate plus tetracycline versus standard treatment (quinine plus tetracycline) for uncomplicated Plasmodium falciparum malaria in Brazil. Am J Trop Med Hyg 1996;54(2):197-202. 
[8] Genovese RF, Newman DB, Brewer TG. Acute high dose arteether toxicity in rats. Neurorotoxicology 1999;20:851-60.

[9] Genovese RF, Newman D, Li Q, Peggins JO, Brewer TG. Dosedependent brainstem neuropathology following repeated arteether administration in rats. Brain Res Bull 1998;45:199-202.

[10] Genovese RF, Newman DB, Petras JM, Brewer TG. Behavioral and neural toxicity of arteether in rats. Pharmacol, Biochem Behav 1998;60(2):449-58.

[11] Genovese RF, Petras JM, Brewer TG. Arteether neurotoxicity in the absence of deficits in behavioral performance in rats. Ann Trop Med Parasitol 1995;89:447-9.

[12] Hien TT, White NJ. Qinghaosu. Lancet 1993;341:603-8.

[13] Kager PA, Schultz MJ, Zijlstra EE, van den Berg B, van Boxtel CJ. Arteether administration in humans: preliminary studies of pharmacokinetics, safety and tolerance. Trans R Soc Trop Med Hyg 1994;88(1): $53-4$.

[14] Karbwang J, Na Bangchang K, Congpoung K, Thanavibal A, Harinasuta T. Pharmacokinetics of oral artesunate in Thai patients with uncomplicated falciparum malaria. Clin Drug Invest 1998;1:37-43.

[15] Koopmans R, Dang Ha L, Dinh Duc D, Kager PA, Khanh NX, Boxtel PJ, DeVries PJ. The pharmacokinetics of artemisinin after administration of two different suppositories to healthy Vietnamese subjects. Am J Med Hyg 1999;60(2):244-7.

[16] Li Q, Peggins JO, Lin AJ, Masonic KJ, Trotman KM, Brewer TG. Pharmacology and toxicology of artelinic acid: preclinical investigations on pharmacokinetics, metabolism, protein and red blood cell binding, and acute and anoretic toxicities. Trans R Soc Trop Med Hyg 1998;92:332-40.

[17] Li Q, Peggins JP, Fleckenstein LL, Masonic K, Heiffer MH, Brewer TG. The pharmacokinetics and bioavailability of dihydroartemisinin, arteether, artemether, artesunic acid and artelinic acid in rats. J Pharm Pharmacol 1998;50:173-82.

[18] Lin AJ, Klayman DL, Milhous WK. Antimalarial activity of new water-soluble dihydroartemisinin derivatives. J Med Chem 1987; 30:2147-50.

[19] Looareesuwan S, Wilairatana P, Vanijanonta S, Pitisuttithum P, Ratanapong Y, Andrial M. Monotherapy with sodium artesunate for uncomplicated falciparum malaria in Thailand: a comparison of 5 - and 7-day regimens. Acta Trop 1997;67(3):197-205.

[20] Meshnick SR, Taylor TE, Kamchonwongpaisan S. Artemisinin and the antimalarial endoperoxides: from herbal remedy to targeted chemotherapy. Microbiol Rev 1996;60:301-15.

[21] Meshnick SR, Yang Y-Z, Lima V, Kuypers F, Kamchonwongpaisan $Y$, Yuthavong Y. Iron-dependent free radical generation from the antimalarial agent artemisinin (qinghaosu). Antimicrob Agents Chemother 1993;37:1108-14.

[22] Mishra SK, Asthana OP, Mohanty S, Patnaik JK, Das BS, Srivastava SK, Satpathy SK, Dash S, Rath PK, Varghese K. Effectiveness of $\alpha$, $\beta$-arteether in acute falciparum malaria. Trans R Soc Trop Med Hyg 1995;89:299-301.

[23] Petras JM, Kyle DE, Gettayacamin GD, Young GD, Bauman RA, Webster HK, Corcoran KD, Peggins JO, Vane MA, Brewer TG. Arteether: risks of two-week administration in Macaca mulatta. Am J Trop Med Hyg 1997;56:390-6.

[24] Phuong CXT, Bethell DB, Phuong TP, Mai TTT, Thuy TTN, Ha NTT, Thuy PTT, Anh NTT, Day NPJ, White NJ. Comparison of artemisinin suppositories, intramuscular artesunate and intravenous quinine for the treatment of severe childhood malaria. Trans R Soc Trop Med Hyg 1997;91(3):335-42.

[25] Price R, Van Vugt M, Phaipun L, Luxemburger C, Simpson J, McGready R, Ter Kuile F, Kham A, Chongsuphajaisiddhi T, White F, Nosten F. Adverse effects in patients with acute falciparum malaria treated with artemisinin derivatives. Am J Trop Med Hyg 1999; 60(4):547-55.

[26] Shmuklarsky MJ, Klayman DL, Milhous WK, Kyle DE, Rossan RN, Ager AL, Tang DB, Heiffer MH, Canfield CJ, Schuster BG. Comparison of $\beta$-artemether and $\beta$-arteether against malaria parasites in vitro and in vivo. Am J Trop Med Hyg 1993;48:377-84.

[27] Titulaer HAC, Eling WMC, Zuidema J. Pharmacokinetic and pharmacodynamic aspects of artelinic acid in rodents. J Pharm Pharmacol 1993;45:830-5.

[28] Tripathi R, Puri SK, Dutta GP. Sodium beta-artelinate - a new potential gameotocytocide. Exp Parasitol 1996;82(3):251-4.

[29] Van Vugt M, Brockman A, Gemperli B, Luxemburger C, Gathmann I, Royce C, Slight T, Looareesuwan S, White NJ, Nosten F. Randomized comparison of artemether-benflumetol and artesunate-mefloquine in treatment of multidrug-resistant falciparum malaria. Antimicrob Agents Chemother 1998;42(1):135-9.

[30] White NJ. Antimalarial drug resistance: the pace quickens. J Antimicrob Chemother 1992;30:571-85. 